REVIEW

\title{
Effect of maternal antibiotics on breast feeding infants
}

\section{J L Mathew}

Postgrad Med J 2004;80:196-200. doi: 10.1136/pgmi.2003.011973

Antibiotic usage is fairly common among breastfeeding mothers and there is potential for transfer to infants through breast milk. While most medicines taken by lactating women cause no harm to their babies, at times it can result in serious consequences. This article reviews the principles governing tranfer of maternal antibiotics to breast milk, its clinical significance, and ways to minimise inadvertent infant exposure.

Correspondence to: Dr Joseph L Mathew, Department of Pediatrics, Advanced Pediatrics Centre, Postgraduate Institute of Medical Education and Research, Chandigarh, 160012, India; jlmathew@hotmail. com or lamat@vsnl.com

Submitted 3 July 2003 Accepted 20 August 2003

....................
B reast feeding is undoubtedly the best form of nutrition for newborns and young infants. Its advantages go far beyond nutritional and anti-infective benefits. Therefore there are almost no contraindications to breast feeding, particularly in developing countries where social, cultural, and economic factors often take precedence over medical issues. Currently, for want of a safer and more cost effective form of providing nourishment, even maternal HIV infection is not an absolute contraindication to breast feeding in these countries. Although the majority of medicines administered to breast feeding mothers cause no harm to babies and there are hardly any contraindications to breast feeding when maternal medication is necessary, initiation and continuation of breast feeding by a mother on antibiotics remains a difficult subject. ${ }^{1}$ On the one hand, the risks have often been overstated, leading to avoidance of a necessary drug or the interruption of breast feeding. At the other extreme, an unrestricted use of drugs can result in a potentially risky situation for the baby, who may absorb therapeutic or even toxic doses while suckling. This article explores the issues to be considered when breast feeding mothers need antibiotic therapy.

\section{PROBLEM STATEMENT AND ISSUES}

For various reasons, antibiotics are one of the commonest groups of drugs prescribed to postpartum mothers. This trend is noticeable both in developing and developed countries. ${ }^{2}$ For example, in Denmark over one third of mothers were prescribed at least one drug after delivery, mostly antibiotics. ${ }^{3}$ In India, a survey revealed that $90 \%$ of mothers delivering in hospitals were prescribed an antibiotic, irrespective of the mode of delivery. An almost equal number attending postnatal clinics also received antibiotics. Of those who delivered at home and were followed up in hospitals, approximately one in every eight mothers was prescribed antibiotics. ${ }^{4}$

Besides the obvious immediate side effects and adverse reactions of inadvertent medication to babies, some drugs also have long term effects on infant growth and development. Premature babies, with more vulnerable renal and hepatic systems, are at greater risk than term babies. With the increasing practice of milk banking for preterm babies, antibiotic administration to even one mother entails a risk to a large number of babies. Antibiotic therapy in this group of mothers has been found to be so common ${ }^{5}$ that at some centres they are excluded from donating milk. Lastly, the ethical issue of administering an unwanted/unwarranted drug to an infant needs to be addressed, particularly in light of the fact that drug testing for safety and efficacy is not carried out in pregnant and lactating women. Thus, when viewed as a whole, administration of drugs to breast feeding mothers is an important issue and merits a closer look.

There are two major questions that arise about possible effects of maternal antibiotics on breast feeding infants. Firstly, can drugs taken by the mother reach the baby through breast milk? Secondly, if yes, can they cause untoward effects, both short term and long term? The first question is relatively easier to answer since transfer of drug molecules to breast milk and absorption by the baby are governed by physical principles. These are often predictable and to an extent can be calculated mathematically. The second issue is more difficult to resolve since there is a paucity of data in human beings (most of the available information is restricted to isolated case reports) and it is inappropriate to extrapolate animal data. Therefore, there is considerable controversy regarding the neonatal considerations of administering antibiotics to breast feeding mothers. ${ }^{67}$

\section{TRANSFER OF ANTIBIOTICS THROUGH BREAST MILK}

The majority of breast milk components are similar to plasma components and theoretically all drugs have the potential of crossing over from maternal plasma into breast milk. Molecular transfer depends on the usual mechanisms governing transfer of molecules across biological membranes, namely, passive diffusion across a concentration gradient, active transport against a concentration gradient and transcellular diffusion, that is, diffusion between adjacent cells (see box 1). Of these, the most important routes are passive diffusion of molecules across the endothelial wall of maternal capillaries and active transport. Transcellular diffusion is almost negligible. In order of relative importance, the factors determining the transfer of antibiotics to

Abbreviations: G6PD, glucose-6-phosphate deficiency; $M / P$ ratio, ratio of concentration in milk to concentration in plasma 
Box 1: Mechanisms of transfer of antibiotics from maternal plasma to breast milk

- Passive diffusion across a concentration gradient.

- Active transport against a concentration gradient.

- Transcellular diffusion.

breast milk include physicochemical properties of antibiotic molecules, individual maternal factors, and infant variations in drug handling capability (box 2).

(A) Physicochemical properties of antibiotic molecules Antibiotics can move across biological membranes only in the unionised state; this movement depends on pKa of the molecule, $\mathrm{pH}$ of plasma, and $\mathrm{pH}$ of breast milk. As for all similar systems, this is governed by the HendersonHasselbach equation: $\mathrm{pH}=\mathrm{pKa}+\log$ (base/acid). Since the $\mathrm{pH}$ of breast milk is lower than plasma $(7.2$ compared with 7.4 of plasma), ${ }^{8}$ weakly acidic drug molecules like sulphonamides and penicillins accumulate in higher concentration in plasma, ${ }^{9}$ whereas weakly basic drugs such as erythromycin, lincomycin, and metronidazole tend to concentrate in milk. Thus the degree of ionisation of a drug determines to a large extent its propensity for being in plasma or breast milk.

Protein binding of antibiotic molecules is the second most important factor determining transfer to milk. Transfer is greatest in the presence of low maternal plasma protein binding. Some drugs may be poorly bound to plasma proteins but get bound to breast milk proteins, which are qualitatively different from plasma proteins. Hence, although such antibiotics reach the infant, they are of little consequence. ${ }^{10-12}$ The half life of antibiotics also determines the concentration in plasma; those with longer half life are more likely to accumulate and be transferred than rapidly cleared molecules. The molecular weight of the drug molecule and lipid solubility are relatively minor contributory factors to the overall process of drug transfer. ${ }^{13}$

It is theoretically possible to calculate the amount of drug that will be transferred from maternal plasma to breast milk; this is conventionally expressed as a ratio of concentration in milk to concentration in plasma ( $\mathrm{M} / \mathrm{P}$ ratio). The $\mathrm{M} / \mathrm{P}$ ratio has been calculated for several of the commonly used drugs ${ }^{12}$; in general, a lower $\mathrm{M} / \mathrm{P}$ ratio implies a lesser amount in milk compared with drugs with a higher ratio. ${ }^{14}$ However, the M/P ratio often does not accurately correlate with the actual amount of a drug in milk. ${ }^{15}$ Its value is improved if calculated from the area under the concentration-time curve of the drug in milk and plasma. The dose of drug received by the suckling infant is then calculated from maternal plasma concentration, area under the curve $\mathrm{M} / \mathrm{P}$ ratio, and the volume of milk ingested. The infant dose can then be expressed as a percentage of the maternal dose. Conventionally, a cut off of $10 \%$ has been used as a rough indicator to guide the prescription of a drug during lactation.

\section{(B) Maternal factors}

As mentioned above, the composition of breast milk and its $\mathrm{pH}$ influence the amount of drug transfer from plasma. There is remarkable interindividual variation in breast milk that can cause different mothers receiving the same dose of antibiotic to transfer different amounts to their babies. In addition, there is also significant intraindividual variation with time, especially in $\mathrm{pH}$. Milk tends to become more acidic with maturity; colostrum may have a pH of around 7.4 whereas transitional milk about 7.05. Likewise, milk from
Box 2: Factors determining infant effects of maternal antibiotics

Physicochemical properties of antibiotic molecules:

- State of ionisation:

- pKa of antibiotic molecule.

- pH of maternal plasma.

- pH of breast milk.

- Affinity for proteins:

- Plasma proteins.

- Milk proteins.

- Half life.

- Molecular weight.

- Lipid solubility.

Individual maternal factors:

- Interindividual variations.

- Intraindividual variations:

- Variation in composition of breast milk with time.

- Variation in $\mathrm{pH}$ of breast milk with time.

- Mammary blood flow.

- Drug metabolism in breast tissue.

\section{Infant factors:}

- Gestational age dependent capacity to handle drugs.

- Gastrointestinal emptying time.

- Gastro-oesophageal reflux.

- Intestinal surface area.

- Duration and frequency of feeding.

- Unpredictable idiosyncratic reactions.

mothers of preterm babies has higher protein content. ${ }^{15}$ Milk expressed from the breast later during suckling (hind milk) has a higher $\mathrm{pH}$ and fat content $\mathrm{t}^{16}{ }^{17}$ and may concentrate fat soluble drugs. It has also been speculated that increased mammary blood flow could result in delivery of a higher quantity of drug into milk, ${ }^{13}$ but its significance has not been established. Breast tissue has the capability to metabolise some drugs, for example sulphonamides, ${ }^{18}$ which may alter the exposure to the suckling infant depending on whether or not the metabolites are active.

\section{(C) Infant factors}

Infants vary in their drug handing capability due to variations in absorption, distribution, metabolism, and elimination of biochemical molecules. Gestational age is probably the most important factor that determines this and premature babies are significantly limited in this regard. This is primarily due to immature renal and hepatic clearance systems. Babies reach adult metabolic capacity only towards the latter part of infancy. Likewise, enzyme mediated metabolic processes such as oxidation, glucuronidation, etc are also incompletely developed in neonates. Other factors that alter absorption include gastrointestinal emptying time, degree of gastro-oesophageal reflux, and relatively smaller intestinal surface area. For these reasons, term babies and "post-conceptionally term" babies behave entirely differently.

Lastly, it must be remembered that side effects and adverse reactions of drugs are not always dose related. There are often 
non-predictable idiosyncratic reactions, whereby two babies receiving the same amount of drug behave differently.

\section{CLINICAL SIGNIFICANCE OF MATERNALLY TRANSFERRED ANTIBIOTICS}

In general, all antibiotics can cause three potential problems for nursing infants. Firstly, they can modify the bowel flora and alter gut defence mechanisms; this can result in diarrhoea and malabsorption of nutrients. Secondly, they may have direct effects that may or may not be dose related. Lastly, and often ignored, is that antibiotics can alter and interfere with microbiological culture resulting in babies being investigated for sepsis. Based on these principles, antibiotics can be categorised under three headings: (1) those that are safe for administration to lactating mothers, (2) those whose effects in breast feeding infants are not known and should be used with caution, and (3) those that are not recommended for breast feeding mothers (box 3 ).

As a general rule, drugs of the same class are expected to behave similarly in infants, though safety of one agent may not always imply the same for other members of the class. It must be emphasised that side effects of maternal medication are often not reported; hence lack of information does not imply safety to the suckling baby. On the other hand, the occurrence of side effects in isolated reports may not necessarily warrant discontinuation of breast feeding or altering the maternal antibiotic prescription. Various academic bodies have tried to compile information about the likely risks to infants; such information may guide decisions about antibiotic use.

Among the antibacterial antibiotics, cephalosporins seem to be the safest class. This generalisation seems to hold for all generations and is irrespective of the route of administration. Although third generation cephalosporins have a greater potential to alter bowel flora and individual agents vary with respect to $\mathrm{M} / \mathrm{P}$ ratio, as a group they are regarded safe.

Box 3: Antibacterial antibiotics and breast feeding

(1) Safe for administration:

- Aminoglycosides.

- Amoxycillin.

- Amoxycillin-clavulanate.

- Antitubercular drugs.

- Cephalosporins.

- Macrolides.

- Trimethoprim-sulphamethoxazole.

(2) Effects not known/to be used with caution:

- Chloramphenicol.

- Clindamycin.

- Dapsone.

- Mandelic acid.

- Metronidazole (low dose).

- Nalidixic acid.

- Nitrofurantoin.

- Penicillins.

- Tetracyclines.

(3) Not recommended:

- Metronidazole (single high dose).

- Quinolones.
Similarly, macrolides are also "safe" antibiotics, though they can alter infants' bowel flora adversely.

For entirely different reasons, another relatively safe class of drugs is the aminoglycoside group. This is because although they are transferred to breast milk, they have poor oral bioavailability and are not absorbed by the infant. However, there is the potential risk of some direct effects as evidenced by a case report of bloody diarrhoea in an infant whose mother was receiving gentamicin and clindamicin. ${ }^{19}$ Both the drugs were found in significant levels in breast milk and diarrhoea subsided when breast feeding was temporarily ceased.

Sulphonamides are excreted into breast milk, but this does not pose a risk for healthy term infants. However, it is recommended that they should be avoided in ill, stressed, or preterm babies as well as infants with hyperbilirubinemia or glucose-6-phosphate deficiency (G6PD) deficiency. Barring these exceptions, the American Academy of Pediatrics considers sulphonamides compatible for use while breast feeding. ${ }^{20}$ The combination with trimethoprim is also safe.

Although penicillins seem to be commonly prescribed to lactating mothers, ${ }^{3}$ there is no hard evidence concerning their safety during breast feeding. They have the potential to alter infant bowel microflora and produce diarrhoea. However, the American Academy of Pediatrics considers them compatible for use during breast feeding. ${ }^{20}$

In contrast, the use of quinolones is not recommended in breast feeding mothers ${ }^{20}$ because of the putative risk of arthropathy noted in animal studies. In addition, phototoxicity has been observed with some quinolones on exposure to ultraviolet light or excessive sunlight. There is only a single report of ciprofloxacin administered by a breast feeding mother and it resulted in no adverse effects to the baby. ${ }^{21}$ However, the manufacturer recommends that 48 hours be allowed to elapse after the last dose of ciprofloxacin before breast feeding is resumed. There is an unusual incident of greenish staining of teeth reported in two neonates treated with ciprofloxacin whose cause could not be located. The clinical significance of this, if any, in lactating mothers is unclear. ${ }^{22}$ Tetracyclines must be avoided during lactation due to possible risks of dental staining and adverse effects on bone development. Metronidazole in a single, high dose is not advised for lactating women, though conventional doses may be used with caution.

A group of drugs that may be of importance, particularly in developing countries, are those used for antitubercular therapy. Unfortunately, not much is known about many of these drugs, though the American Academy of Pediatrics categorises them as compatible with breast feeding. However, isoniazid has the potential for interference with nucleic acid metabolism and can cause hepatotoxicity in the infant. ${ }^{23}{ }^{24}$ Hence, in mothers taking isoniazid, it is suggested that infants be regularly examined for peripheral neuritis and hepatotoxicity. Streptomycin does not carry the risk of ototoxicity in suckling babies because it is poorly absorbed from the gut. There are few data available on ethambutol, rifampicin and pyrazinamide, but their use is regarded as compatible with breast feeding. ${ }^{20}$

Among the antiviral antibiotics, acyclovir is transferred in significant amounts to breast milk, but its poor absorption through the oral route makes it compatible with breast feeding. Since acyclovir is the active metabolite of valacyclovir, the latter also is regarded safe, although no human studies are available. The current recommendation of the Centers for Disease Control and Prevention restricts HIV infected women in developed countries from breast feeding; hence there is no information on the infant effects of maternal antiretroviral antibiotics. Likewise, there is no information available with regard to ganciclovir. Although 


\section{Box 4: Other antibiotics and breast feeding}

\section{(1) Antiviral}

- Compatible with breast feeding:

- Acyclovir.

- Amantadine.

- Valacyclovir.

- Effects during breast feeding are not known:

- Antiretrovirals.

- Famciclovir.

- Foscarnet.

- Ganciclovir.

\section{(2) Antifungal}

- Compatible with breast feeding:

- Ketoconazole.

- Effects during breast feeding are not known:

- Amphotericin.

- Fluconazole.

- Flucytosine.

- Itraconazole.

\section{(3) Antimalarial}

- Compatible with breast feeding:

- Chloroquine.

- Hydroxychloroquine.

- Quinine.

- Effects during breast feeding are not known:

- Mefloquine.

- Pentamidine.

- Proguanil.

- Primaquine.

- Pyrimethamine.

(4) Antihelminthic

- Effects during breast feeding are not known:

- Mebendazole.

- Pyrantel pamoate.

- Praziquantel.

- Quinacrine antihelminth.

- Thiabendazole.

- Piperazine.

famciclovir and foscarnet are detectable in significant amounts in breast milk, there are no data on the effects in sucking infants.

Amphotericin B is an antifungal agent that has been well studied in infants, however the effects on maternally transferred drug are not known. Similarly, fluconazole is transferred to milk, but effects on babies are not documented. Flucytosine, itraconazole, ketoconazole, and terbinafine are other antifungal antibiotics that are transferred in varying amounts to breast milk, but their potential effects on nursing infants are unknown.

Among the antimalarials, chloroquine, hydroxychloroquine, and quinine are found in variable quantities in breast milk, but all three are regarded as compatible with breast feeding. However, quinine is advised to be withheld in mothers with G6PD deficient infants. Insufficient information is currently available to make specific recommendations about the use of pentamidine, proguanil, primaquine, and mefloquine. Pyrimethamine can result in serious side effects such as bone marrow suppression; however it is regarded as compatible with breast feeding. ${ }^{20}$

The usual antihelminthic drugs include mebendazole, pyrantel pamoate, praziquantel, thiabendazole, and piperazine. No information is available on the infant effects of these drugs in lactating mothers; however, mebendazole has been shown to decrease milk output in some women. Box 4 shows the status of these antibiotics during breast feeding.

\section{MINIMISING THE RISKS OF MATERNAL ANTIBIOTICS IN INFANTS}

Although the risk of transfer of antibiotics through breast milk can never be eliminated altogether, it can be minimised to a great degree. The cornerstones of this depend on awareness of untoward effects of maternal antibiotics, judicious prescription to lactating mothers, strategies to limit absorption by suckling infants, and monitoring of nursing babies (box 5).

Before prescription of maternal antibiotics, the physician needs to be certain that there is a real need for them. The likely benefits have to be weighed against the risk of not breast feeding or the potential risk of exposing the infant to undesirable drug effects. In each situation where antibiotics are considered, it is appropriate to discuss the risks with mothers/parents and educate them about the potential risks to breast feeding babies. This is particularly important because while risks of antibiotic use during pregnancy are

\section{Box 5: Minimising the exposure of infants to} undesirable maternal antibiotic effects

- Strategy 1: Judicious prescription of antibiotics to lactating mothers:

- Is antibiotic necessary?

- If yes, does it have to be administered systemically?

- Weigh likely benefits and risks.

- Discuss benefits and risks with mother/parents.

- Strategy 2: Decreasing transfer of antibiotics to breast milk:

- Prescription of antibiotics that have poor oral bioavailability.

- Use of topical/local antibiotics where feasible.

- Preferential prescription of antibiotics with rapid clearance from plasma.

- Feeding the baby immediately before antibiotic dose.

- Dose modification of maternal antibiotic.

- Bottle feeding at time of peak plasma concentration of antibiotic.

- Omission of breast feeding is a last resort.

- Strategy 3: Monitoring the infant:

- Watch for untoward/unexpected/unexplained effects.

- Confirm if this could be related to maternal antibiotic therapy.

- Monitoring of serum levels. 
well recognised, this is not always so during lactation. A drug that is safe for use during pregnancy may not be safe for the nursing infant. ${ }^{25}$

Some of the strategies to reduce infant exposure to maternal antibiotics include prescribing antibiotics that are poorly absorbed orally and avoiding breast feeding during peak maternal serum concentration. In some situations, it may be feasible to give mothers topical or local antibiotic therapy, reducing the chances of infant exposure. Staggering the schedule so that the baby is fed immediately before the dose of antibiotic is also a simple yet useful strategy. In some instances, it may be feasible to choose antibiotics with more rapid clearance in preference to those with longer half life; the former will be transferred less to breast milk than the latter. ${ }^{11}$ Where it is feasible, it may be useful to suggest alternating breast feeding with bottle feeding for brief durations. If antibiotics are absolutely unavoidable and premature babies are at risk of exposure, it may be prudent to alter dosage to avoid drug accumulation and toxicity in infants. ${ }^{25}$ When all these measures fail, only then should temporary omission of breast feeding be considered as a last resort.

Lastly, it is always wise to monitor the baby closely for any untoward or unexplained effect that can be attributed to maternal antibiotics. These may be non-specific such as lethargy, poor feeding, etc, or specific side effects like diarrhoea. In some cases, it may even be necessary to measure drug levels in infants' serum. Regularly updated information is available from a variety of academic sources, whereby it can be confirmed whether effects occurring in infants are related to maternal antibiotics or not. Such helplines are useful and must be accessed at the first sign of doubt.

\section{CONCLUSION}

While there is no ambiguity that breast feeding is the best form of nutrition to infants, certain issues need to be addressed when the mother is on short or long term antibiotic therapy. These encompass clinical issues, sociocultural aspects, and may also include ethical considerations. Based on pharmacokinetic studies and case reports published, it is now possible to determine the effects of several maternal antibiotics on suckling infants. It may be possible to minimise exposure of babies to untoward effects of antibiotics through awareness, judicious prescription, and modifications in antibiotic schedule. These issues need consideration in order to enable physicians and mothers to make the best possible decision with respect to the baby's interests.

\section{REFERENCES}

1 Chin KG, Mactal-Haaf C, McPherson CE. Use of anti-infective agents during lactation: part 1 -beta-lactam antibiotics, vancomycin, quinupristindalfopristin, and linezolid. J Hum Lact 2000;16:351-8.

2 Thomas M, Jairaj P, Mathew LG. A prospective study in a southern Indian hospital on the prescription of medication during the lying in period following childbirth. Soz Praventivmed 1994;39:273-9.

3 Olesen C, Steffensen FH, Nielsen GL, et al. Drug use in first pregnancy and lactation: a population based survey among Danish women. The EUROMAP group. Eur J Clin Pharmacol 1999;55:139-44.

4 Uppal R, Karmakar S, Singh MM, et al. Prescription drug use in lactating mothers: an experience at a referral hospital and in a community in India. Int J Clin Pharmacol Ther Toxicol 1993;31:93-5.

5 Hoppu K, Kettunen K, Remes R. Maternal drug treatment and human milk banking. Int J Clin Pharmacol Ther 1994;32:488-90.

6 Scott A, Forsyth S. Breast feeding and antibiotics. Mod Midwife 1996;6: 14-6.

7 Bowes WA Jr. The effects of medications on the lactating mother and her infant. Clin Obstet Gynecol 1980;23:1073-80.

8 Rasmussen F. The mechanisms of drug secretion into milk. In: Galli G, Jacini G, Pecile A, eds. Dietary lipids and postnatal development. New York: Raven Press, 1973:231.

9 Bourget P, Quinquis-Desmaris V, Fernandez H. Ceftriaxone distribution and protein binding between maternal blood and milk postpartum. Ann Pharmacother 1993;27:294-7.

10 Miller GE, Banerjee NC, Stowe CM. Diffusion of certain weak organic acids and bases across the bovine mammary gland membrane after systemic administration. J Pharmacol Exp Ther 1967;157:245.

11 Miller GE, Peters RD, Engebretsen RV. Passage of pentobarbital and phenobarbital into bovine and caprine milk after systemic administration. J Dairy Sci 1967;50:7692.

12 Taddio A, Ito S. Drug use and lactation. In: Koren G, eds. Maternal-foetal toxicology. 2nd Ed. New York: Dekker, 1994.

13 Wilson JT, Brown RD, Cherek DR. Drug excretion in human breast milk: principles, pharmacokinetics and projected consequences. Clin Pharmacokinet 1980;5:1.

14 Wojnar-Horton RE, Hackett LP, Dusci U. Distribution and excretion of sumatriptan in human milk. Br J Pharmacol 1996;41:217.

15 Chung AM, Reed MD, Blumer JL. Antibiotics and breast-feeding: a critical review of the literature. Paediatr Drugs 2002;4:817-37.

16 Morris FH, Brewer ED, Spedale SB. Relationship of human milk pH during course of lactation to concentrations of citrate and fatty acids. Pediatrics 1986;78:458.

17 Prentice A, Prentice AM, Whitehead RG. Breast-milk fat concentrations of rural African women. I. Short term variations within individuals. $\mathrm{Br} J$ Nutr $1981 ; 45: 483$.

18 Rasmussen F, Linzell JL. The acetylation of sulfanilamide by mammary tissue of lactating goats. Biochem Pharmacol 1967;16:918.

19 Mann CF. Clindamycin and breast-feeding. Pediatrics 1980;66:1030-1.

20 Committee on drugs, American Academy of Pediatrics. The transfer of drugs and other chemicals into human milk. Pediatrics 1994;93:137-50.

21 Gardner DK, Gabbe SG, Harter C. Simultaneous concentrations of ciprofloxacin in breast milk and in serum in mother and breast-fed infant. Clin Pharm 1992;11:352-4

22 Lumbiganon $\mathbf{P}$, Pengsaa K, Sookpranee T. Ciprofloxacin in neonates and its possible effect on the teeth. Pediatr Infect Dis J 1991;10:619-20.

23 Snider DE Jr, Powell KE. Should women taking antituberculous drugs breastfeed? Arch Intern Med 1984;144:589-90.

24 Berlin CM Jr, Lee C. Isoniazid and acetylisoniazid disposition in human milk, saliva and plasma. Fed Proc 1979;38:426.

25 Spencer JP, Gonzalez LS 3rd, Barnhart DJ. edications in the breast-feeding mother. Am Fam Physician 2001;64:119-26. 